\title{
Dynamic effects in the scattering of electrons by small clusters of atoms
}

\author{
L. S. Bartell \\ Department of Chemistry, University of Michigan, Ann Arbor, Michigan 48109 \\ B. Raoult and G. Torchet \\ Groupe des Agrégats Moléculaires, Laboratoire de Diffraction Electronique*-Université de Paris Sud. \\ 91405 Orsay Cedex. France \\ (Received 19 January 1977)
}

\begin{abstract}
Dynamic scattering corrections were calculated for $40 \mathrm{kV}$ electrons diffracted by randomly oriented fcc clusters of argon and of gold atoms ranging in size from 13 to 135 atoms. Computations were carried out according to several variants of two limiting theoretical approaches, namely, the direct summing up of atomic contributions calculated through single-single and single-double scattered waves by modifications of Glauber theory, and the extrapolation to limitingly small crystallites of conventional dyramic theory in the Blackman and Fujimoto formulations. For the small clusters studied, integrated intensities of diffraction rings (through single-double scatterings) calculated for three dimensional crystallites differ insignificantly from Glauber theory intensities calculated by projecting atomic potential energies onto a plane perpendicular to the mean direction of the incident and scattered wave vectors. The fractional dynamic correction increases with cluster size very nearly as $N^{2 / 3}$ in both the Glauber and Blackman-Fujimoto limiting treatments. For crystalline clusters $8-20 \mathrm{~A}$ in diameter, the dynamic effect calculated by summing single-double scatterings is an order of magnitude larger than that according to Blackman-Fujimoto theory. For argon clusters the dynamic effect is not serious; but according to our direct sums, dynamic corrections for 16 A spheres of gold are surprisingly large, exceeding $25 \%$ for 111 reflections. Since the direct sums have been verified experimentally for several vapor-phase molecules, the present work indicates that, in the limit of very small scatterers, extrapolations from conventional two-beam dynamic theory may seriously underestimate the magnitude of dynamic effects.
\end{abstract}

\section{INTRODUCTION}

Recent advances in the study of extremely small nucleation clusters by electron scattering technique $\mathrm{s}^{1-3}$ have stimulated renewed interest in the theory of dynamic scattering. Subtle deviations have been observed between experimental scattered intensities and intensities calculated for idealized structures according to kinematic theory. While a persuasive case can be made that thesedeviations reveal differences between the packing of atoms in small aggregates and the packing in bulk crystals, ${ }^{1}$ it is prudent to assess the extent to which dynamic scattering may distort intensities and becloud interpretations. Despite the widespread feeling ${ }^{4}$ that dynamic effects can be disregarded for electrons in the $50 \mathrm{kV}$ range scattered by randomly oriented crystallites less than 30-50 $\AA$ across, clear and significant dynamic manifestations are apparent in the scattering patterns of single gas-phase molecules containing no more than six or seven atoms. ${ }^{5-7}$ Therefore it is not obvious that dynamic scattering can be safely neglected in the scattering by clusters. Indeed, we shall show that in the limit of small clusters, dynamic effects are more than an order of magnitude larger than suggested by conventional two-beam theory!

Conventional dynamic scattering theory ${ }^{8}$ has been successful in accounting for observations with relatively large crystallites ${ }^{9}$ (well in excess of $50 \AA$ ), but difficulties are encountered in applying it to very small systems. Blackman ${ }^{10}$ was the first to extend Bethe's theory ${ }^{8}$ to the treatment of intensities of diffraction rings obtained from thin polycrystalline films. His calculations were applicable strictly only to scattering by homogenous parallel-sided slabs. More recently,
Fujimoto $^{11}$ developed Fengler's theory ${ }^{12}$ employing higher Born approximations and adapted it to spherical crystallites embedded in parallel-sided foils. His results were similar to those of Blackman but characterized more precisely the relation between crystallite size, ring intensities, and ring breadths. Nevertheless, not even Fujimoto's elegant approach yielded a rigorous treatment for clusters of arbitrary shape and structure, and the extrapolation all the way down to systems with relatively small numbers of molecules is uncertain. Therefore, it is fortunate that theories developed to treat dynamic scattering by gas-phase molecules ${ }^{6,13-15}$ can be reduced to a form tractable enough to treat randomly oriented clusters of arbitrary shape and structure containing 100 atoms or more. In the next section we outline the approach.

\section{THEORY}

\section{A. Modified eikonal approximation}

Various treatments of intramolecular multiple scattering of electrons by gas molecules have been published. ${ }^{6,13-20}$ An approach which yielded comparatively simple analytical expressions for intensities averaged over all molecular orientations ${ }^{6,13}$ is the Glaube ${ }^{21}$ highenergy approximation. In this eikonal ${ }^{22}$ approach, which is closely related to one developed by Moliere, ${ }^{23}$ the scattering factor for an electron with incident momentum $\hbar k$ experiencing a static potential energy $V(r)$ in a target molecule is

$$
f(s) \approx-(i k / 2 \pi) \int d^{2} p \exp (i \mathrm{~s} \cdot \mathrm{p})\{\exp [i \chi(\mathrm{p})]-1\},
$$

where the momentum transfer $\hbar \mathbf{s}$ is $\hbar\left(\mathbf{k}-\mathbf{k}^{\prime}\right)$, with $\mathbf{k}^{\prime}$ 
representing the scattered wave vector, and the magnitude of $\mathrm{s}$ is $2 k \sin (\theta / 2)$, where $\theta$ is the scattering angle. The semiclassical phase shift $\chi(p)$ is given by

$$
\chi(\mathbf{p})=-(\hbar v)^{-1} \int_{\infty}^{\infty} d z^{\prime} V\left(\mathbf{p}+\mathbf{K} z^{\prime}\right)
$$

where $\mathrm{K}$ is a unit vector along the $z^{\prime}$ axis, $\mathrm{p}$ is the impact parameter perpendicular to $K$, and the $z^{\prime}$ axis is chosen in the simplest variant of Glauber theory to be parallel to $\mathbf{k}$ or, in a modified variant, to be parallel to $\mathbf{k}+\mathbf{k}^{\prime}$. If the potential energy is taken to be the sum $\sum_{i} V_{i}\left(\mathrm{p}_{i}+\mathrm{K} z_{i}^{\prime}\right)$ of potential energies associated with atoms $i, j, k, \ldots, \mathrm{Eq}$. (1) can be expressed as

$$
f(\mathbf{s})=\sum_{i} f_{i} \exp \left(i \mathbf{s} \circ \mathrm{p}_{i}\right)+\sum \sum_{i<j} f_{i j}+\sum \sum_{i<j<k} \sum_{i j k}+\cdots,
$$

in which the sums represent single, double, triple, etc., scattering. The inequalities in Eq. (3) signify that intra-atomic multiple scattering is built into the individual atomic scattering factors $f_{i}(s)$ which are derivable from Eqs. (1) and (2) with $V=V_{i}$. Atomic scattering factors, then, are complex functions expressible as

$$
f_{i}(s)=\left|f_{i}(s)\right| \exp i \eta(s) \text {. }
$$

Scattered intensities from randomly oriented molecules are proportional to the orientationally and vibrationally ave raged differential cross sections

$$
\begin{aligned}
\langle d \sigma / d \Omega\rangle & =\left\langle|f(\mathbf{s})|^{2}\right\rangle=\sum_{i} \sum_{j} f_{i} f_{j} j_{0}\left(s r_{i j}\right) \exp \left(-l_{i j}^{2} s^{2} / 2\right)+\sum_{i} \sum_{j} \sum_{k} 2 \operatorname{Re}\left\langle f_{k}^{*} \exp \left(-i \mathbf{s} \cdot \mathbf{p}_{k}\right) f_{i j}\right\rangle+\cdots \\
& =\sum_{i} \sum_{j}\langle d \sigma / d \Omega\rangle_{i j}+\sum_{k}\left[\sum_{i\langle j}\langle d \sigma / d \Omega\rangle_{k, i j}\right]+\cdots
\end{aligned}
$$

corresponding to single-single, single-double, etc., scatterings, where $j_{0}\left(s r_{i j}\right)$ is a spherical Bessel function, $r_{i j}$ is the distance between atoms $i$ and $j$, and $l_{i j}^{2}$ is the mean-square amplitude of vibration of $i$ with respect to $j$. The single-double scattering from a given $k$, ij fragment is very nearly ${ }^{6,13}$

$$
\langle d \sigma / d \Omega\rangle_{k, i j}=-\left(2 / k r_{i j}^{2}\right) J_{0}\left(s r_{k}\right)\left|f_{i}\left(s_{i}\right)\right|\left|f_{j}\left(s_{j}\right)\right|\left|f_{k}(s)\right| \sin \left[\eta_{i}\left(s_{i}\right)+\eta_{j}\left(s_{j}\right)-\eta_{k}(s)+H_{i j}\right] \exp \left(-l_{\perp}^{2} s^{2} / 2\right)_{k, i j},
$$

where $H_{i j}$ is zero in the pure eikonal approximation, and $l_{1}^{2}$ is a mean-square amplitude of vibration defined in Ref. 6. The other quantities can be understood in terms of the following construction. Pass a line through atom $k$ that intersects at right angles a line through atoms $i$ and $j$. Let $r_{i}, r_{j}$, and $r_{k}$ be the distances of atoms $i, j$, and $k$ from this intersection, point 0 . Then the quantities

$$
\begin{aligned}
& s_{i}=\left(r_{j} / r_{i j}\right) s, \\
& s_{j}=\left(r_{i} / r_{i j}\right) s,
\end{aligned}
$$

and $s r_{k}$ in the argument of the cylindrical Bessel function $J_{0}\left(s r_{k}\right)$ are defined. The above equations can be applied to any structure with specified atomic positions and amplitudes of thermal motion.

Inasmuch as eikonal theory is applicable to the range

$$
s^{2} \ll k / d \text {, }
$$

where $d$ is the distance over which the potential energy is appreciable, this inequality ${ }^{21}$ is surely violated in the range of scattering angles of interest if $d$ is a distance in a crystallite. The aspect of the eikonal theory leading to the restrictions in Eq. (7) is the assumption that electron trajectories in the target are all parallel to vector $\mathbf{K}$. Therefore, Glauber theory has been modified in its treatment of $f_{i j}$ to allow waves scattered by the first atom, $i$, enountered to propagate in the space between the first scattering and a subsequent scattering by atom $j .^{13}$ In the case where $r_{i j}$ is large compared with the scattering length of atom $i$, the resultant modification of Eq. (6) is very simple, and consists of replacing $H_{i j}=0$ with a new phase shift ${ }^{13}$ of

$$
H_{i j}=\left(r_{i} r_{j} / 2 k r_{i j}\right) s^{2}
$$

if intersection 0 is between atoms $i$ and $j$, and

$$
H_{i j}=-\left(r_{i} r_{j} / 2 k r_{i j}\right) s^{2}
$$

if the intersection is outside atoms $i$ and $j$. Approximations that have entered $\mathrm{Eq}$. (6) with its phase correction of Eq. (8) restrict the applicability to values of $s$ that are smaller than $\left(\pi k^{2} r_{i j} / r_{l} r_{k}\right)^{1 / 3}$, where $r_{l}=r_{i}$ or $r_{j}$. This restriction is substantially less severe than that of Eq. (7).

A Born series can be expressed in the form of $\mathbf{E q}$. $(3)^{14,24}$ and (if it converges properly) it represents an arbitrarily exact solution of Schrödinger's equation for potential scattering (elastic scattering, neglecting effects of polarization and inelastic scattering). The approach in the present paper assumes that the modified Glauber solution adequately represents single and double scattering, and that higher order interactions than double scattering may be neglected. This surely cannot be true for crystallites of any great size, but it is reasonable to regard Eqs. (6) and (8) as expressing the magnitude of dynamic scattering in the limit of small clusters of atoms. The virtue of the equations is that they can be applied to clusters of arbitrary shape and structure, with any thermal motion it is desired to consider, simply by regarding a cluster as a giant molecule. Since a sum over all combinations of threeatom fragments $k, i j$, is required, the number of terms to be handled increases rapidly as the number of atoms, $N$, increases. Only nonlinear $k-i-j$ groupings will contribute oscillatory single-double interference terms to the diffraction pattern. In the case of $N=13$ atoms 
TABLE I. Cluster diameters in $\AA$.

\begin{tabular}{lllll}
\hline \hline$N$ & 13 & 55 & 87 & 135 \\
\hline$D(\mathrm{Ar})$ & 9.8 & 15.8 & 18.4 & 21.3 \\
$D(\mathrm{Au})$ & 7.5 & 12.1 & 14.1 & 16.4 \\
\hline \hline
\end{tabular}

packed in an fcc array, there are 840 such groups to be included, many of which are equivalent. If $N$ is increased to 135 (the maximum value we have treated to date), there are more than one million $k-i-j$ groups, making it essential to use an efficient computer code to search for, compute, and store the desired interactions. Our program required $45 \mathrm{sec}$ for $N=55$ and 10 min for $N=135$ on a Univac 1110 .

\section{B. Blackman-Fujimoto theory}

We shall review the conventional dynamical theory for calculating intensities of Debye-Scherrer rings in electron diffraction only in sufficient detail to specify the equations we used in computations to compare theories. Blackman's integ rated intensities scattered by the $h k l$ planes in a polycrystalline film of thickness $H$ can be expressed ${ }^{10}$

$$
I_{h k l}^{D} \approx I_{h k l}^{K}\left[1-\left(A^{2} / 3\right)+\left(A^{4} / 20\right)+\cdots\right]
$$

if $H$ is small enough, where $I_{h k l}^{D}$ is the averaged intensity calculated by dynamic theory, $I_{h b l}^{K}$ is the corresponding quantity according to kinematic theory, and

$$
A=v_{h k t} H / 2 k^{\prime} \cos \theta_{2},
$$

where $\left(\hbar^{2} / 2 m e\right) v_{h h t}$ is the Fourier coefficient of the potential energy of the electron in the film, $k^{\prime}$ is the wave vector magnitude of the electron in the film, and $\theta_{2}$ is the (fairly small) angle between the directions of the reflected wave and the normal to the surface of the crystal. In the present study of fcc crystallites with four atoms per cell,

$$
v_{h k t}=-4\left(4 \pi / a_{0}^{3}\right)\left|f\left(s_{h k l}\right)\right|
$$

where $a_{0}$ is the lattice parameter, $f$ is the electron atomic scattering factor, and $s_{h k l}$ is the value of the angular variable $s$ at which the $h k l$ plane reflects. In the remainder of the paper we shall take $k^{\prime} \approx k$ and $\cos \theta_{2}$ $\approx 1$ with a loss of accuracy small compared with the disparity between the modified eikonal and conventional dynamic theories.

Fujimoto's extension ${ }^{11}$ of Fengler's theory ${ }^{12}$ to the case of randomly oriented spherical crystallites of diameter $D$ embedded in a film ultimately reduces to an equation very similar to that of Blackman, namely,

$$
I_{h h l}^{D} \approx I_{h k t}^{\pi}\left[1-\left(A^{2} / 3\right)+1.2\left(A^{4} / 20\right)+\cdots\right],
$$

where $A$ has the same meaning as before if the association $D=1.291 H$ is made to relate the Fujimoto diameter to the Blackman thickness. Fujimoto also derives an expression for the integral line breadth of the DeybeScherrer ring which deviates significantly from the kinematic breadth as parameter $A$ increases. While the ring intensity profiles are calculated directly in the present research, our values of $A$ are too small to af- ford a useful comparison of line breadths with the Fujimoto theory. Indeed, the precise meaning of individual ring intensities $I_{h k t}$ becomes muddy with our exceedingly small crystallites.

\section{COMPUTATIONS FOR fCc CLUSTERS}

Clusters of argon or gold atoms in cubic closest packing geometries were selected to serve as scatterers in direct calculations of differential cross sections for $40 \mathrm{kV}$ electrons according to Eqs. (5) and (6). Structural units with $N=13,55,87$, and 135 atoms per cluster were constructed to have approximately spherical shapes. In the $N=13$ case, 12 atoms were close packed in the first coordination sphere of the central atom. In the $N=55$ case, an additional 42 atoms were packed in the second coordination sphere. In the $N=87$ and 135 cases, 32 or 80 atoms were added to the next coordination sphere. Cluster diameters, $D$, were calculated for use in Fujimoto's dynamic scattering formula, Eq. (12), by relating the cluster volume $V_{c}$ or (volume per cell) $\times$ (atoms) $\div$ (atoms per cell) to the diameter via

$$
V_{c}=(4 \pi / 3)(D / 2)^{3}=a_{0}^{3} N / 4 \text {. }
$$

Results are shown in Table I.

In the calculation of scattered intensities by means of Eq. (6), the partial wave values of $|f(s)|$ and $\eta(s)$ tabulated by Schafer, Yates, and Bonham ${ }^{25}$ were employed rather than atomic scattering factors derived from $\mathrm{Eq}$. (1) but, as shown by Yates and Tenney, ${ }^{16}$ this makes no perceptible difference for $40 \mathrm{kV}$ electrons. The same scattering factors were used in Eq. (11) in the course of applying Fujimoto's theory. For sake of argument, it was assumed that all amplitudes of vibration $l_{i j}$ and $l_{1}$ were $0.2 \AA$. Since electron diffraction patterns are almost invariably taken with the aid of rotating sectors to screen out differentially the excessively strong scattering at small angles, we have multiplied our calculated differential cross sections by a factor of $s^{3}$ to simulate the effect of a standard sector.

\section{RESULTS}

Results of applying the modified eikonal theory expressed in Eqs. (5), (6), and (8) to $40 \mathrm{kV}$ electrons scattered by argon and by gold clusters are shown in Figs. 1 and 2 . In the se illustrations the smooth atomic background corresponding to the diagonal terms in Eq. (5) has been deleted and the single-single (kinematic, $I_{2}$ ) and single-double (dynamic, $I_{3}$ ) interference terms are shown with $I_{3}$ augmented by constant factors for simplicity of representation. Line shapes as well as intensities of the Debye-Scherrer rings can be seen. As $N$ gets larger, the rings $\left(I_{2}\right)$ and ring corrections $\left(I_{3}\right)$ become narrower and ultimately, in the limit of large $N$, they would show up as sharp spikes on a smooth background. In order to compare ring intensities calculated by the modified Glauber theory ${ }^{9}$ with the integrated intensities of Fujimoto, ${ }^{11}$ it is helpful to draw smooth backgrounds representing baselines for the rings in the diagrams of ring profiles. In the case of the kinematic curves, $I_{2}$, the baselines should pass smoothly below the interference oscillations. For the 


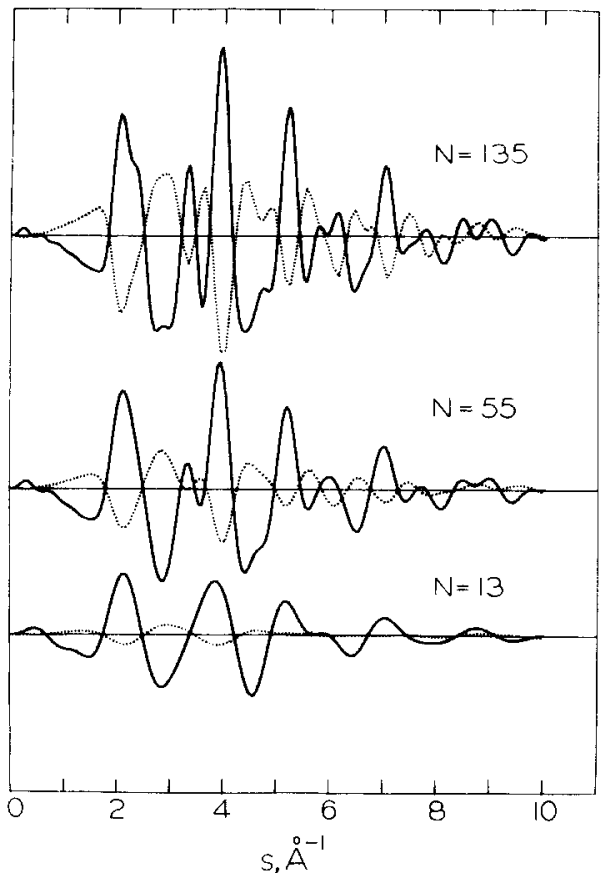

FIG. 1. Diffraction patterns $s^{3} I(s)$ of $40 \mathrm{kV}$ electrons scattered by argon clusters. Kinematic interference intensities (solid curves). Dynamic single-double scattering corrections (dotted curve) calculated according to Eqs. (6) and (8). Dynamic corrections are multiplied by a factor of 20 relative to kinematic intensities for clarity.

dynamic corrections, $I_{3}$, the "baselines" lie above the oscillations because the dynamic corrections are negative to conserve flux. Drawing the backgrounds is somewhat subjective, and the more so as $N$ decreases, but the uncertainty is small compared with the discrep-

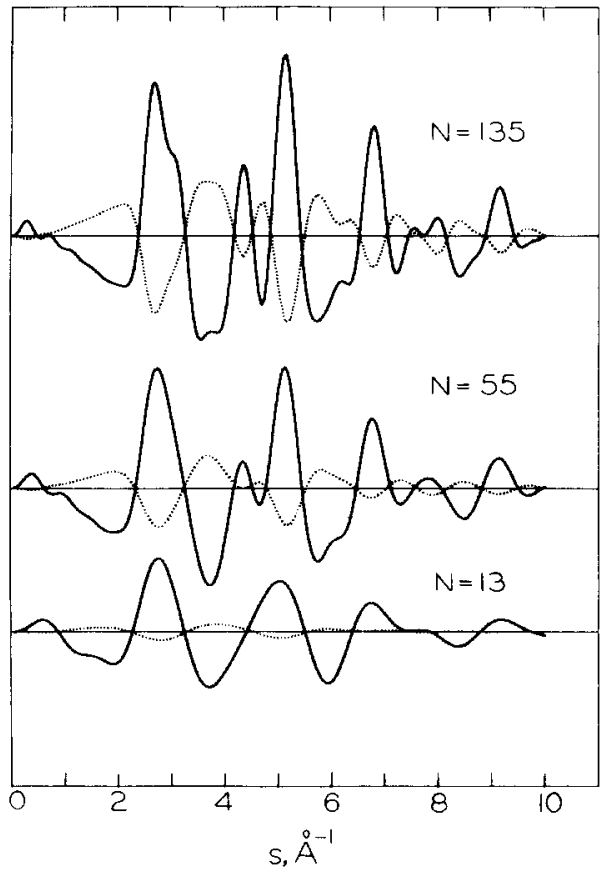

FIG. 2. Diffraction patterns from gold clusters; details same as in Fig. 1 except that dynamic corrections are multiplied by a factor of only 2 .

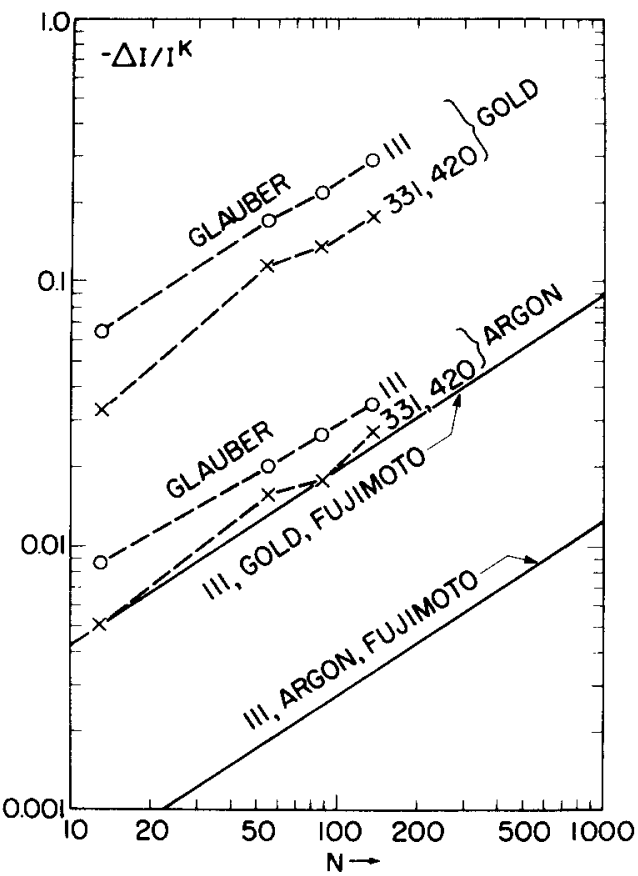

FIG. 3. Fractional dynamic corrections for gold and argon clusters as a function of cluster size ( $N=$ atoms per cluster). Points and crosses, single-double scattering sums calculated for 111 and for 331,420 reflection peaks, respectively. Solid lines, corrections for 111 reflections calculated according to Fujimoto's theory.

ancy between Glauber theory extended to large systems and Fujimoto theory extrapolated to small $N$. Since the $I_{2}$ and $I_{3}$ ring breadths are comparable, we have assumed for simplicity that integrated ring intensities are proportional to the differences between the curves of Figs. 1 and 2 and their smooth baselines (not illustrated).

Perhaps the simplest comparison between the modified eikonal theory and the conventional dynamic theory for crystals is given by $\Delta I / I$, the ratio of dynamic ring corrections to the kinematic ring intensities [i.e., $-\left(I_{3}\right.$ $\left.-I_{3}^{B}\right) /\left(I_{2}-I_{2}^{B}\right)$, or $-\left(I_{h k l}^{D}-I_{h k l}^{K}\right) / I_{h k l}^{K}$, where $I^{B}$ represents the baseline intensity]. How these quantities vary with $N$ and with scattering angle is shown in Figs. 3 and 4.

\section{DISCUSSION}

The most important results of this work can be summarized in six points:

(1) For clusters in the range $13 \leqslant N \leqslant 135$, the difference between the dynamic corrections of Glauber theory [Eq. (6) with all $H_{i j}=0$ ] and modified Glauber theory [Eq. (6) with $H_{i j}$ according to Eq. (8)] is minor, notwithstanding the large differences for many $k$, ij components as illustrated in Fig. 2 of Ref. 13. There are small differences in detail, but magnitudes differ too little to warrant plots of each. In the following, then, the designation Glauber theory pertains to any variant discussed above.

(2) For small clusters, the fractional dynamic cor- 


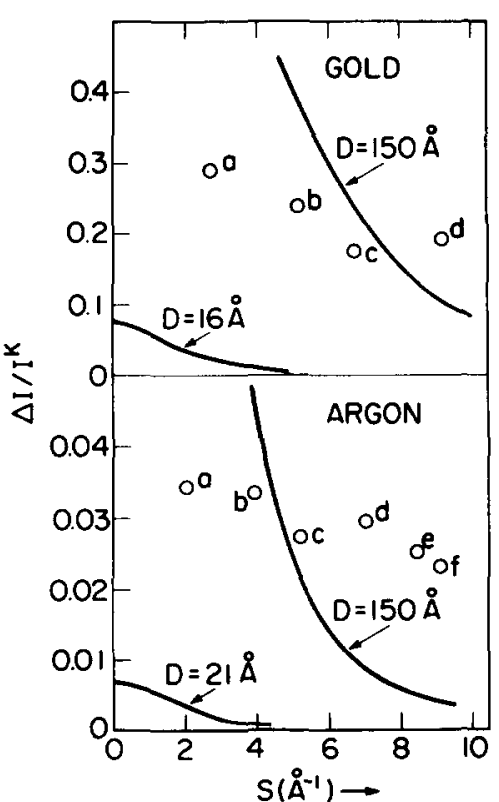

FIG. 4. Fractional dynamic corrections as a function of scattering angle, for gold and argon clusters. Solid curves, corrections calculated according to Fujimoto's theory for clusters of 135 atoms ( $16 \AA$ for $\mathrm{Au}, 21 \AA$ for $\mathrm{Ar}$ ) and clusters $150 \AA$ in diameter. Points, single-double scattering sums calculated for clusters of 135 atoms at the following strong interference peaks: (a) 111 ; (b) 311,222 ; (c) 331,420 ; (d) $531,442,600$; (e) $551,711,640$; (f) 553,731 .

rection $\Delta I / I$ varies with cluster size very nearly as $N^{2 / 3}$ in both Glauber and Blackman-Fujimoto theory.

(3) For crystalline clusters in the range of 8-20 , the fractional dynamic correction $\Delta I / I$ for 111 reflections is an order of magnitude larger according to Glauber than according to Fujimoto theory!

(4) As the Miller indices increase, the disparity between Glauber and Fujimoto theory, already enormous for 111 reflections, increases. That is, for small crystallites $\Delta I / I$ falls off as $|f(s)|^{2}$ according to Blackman and Fujimoto, and $|f(s)| \approx[Z-F(s)] / s^{2}$ decreases rapidly with $s$. By contrast, the Glauber $\Delta I / I$ falls comparatively slowly as $s$ increases.

(5) Dynamic corrections for argon crystallites with $N \approx 100$ are only $3 \%$ or less according to Glauber theory. As the crystallites increase in size, the scattering conditions probably conform more and more closely to those of conventional dynamic theory which predicts dynamic effects of $3 \%$ or less at $N=10000$. Therefore, dynamic distortions can be neglected with little risk when interpreting electron diffraction patterns from nucleation clusters in terms of cluster structure with the aid of kinematic theory.

(6) By contrast, dynamic corrections for gold crys tallites a mere $16 \AA$ in diameter already exceed $25 \%$ according to Glauber theory. Contrary to some published speculations ${ }^{26}$ that Blackman theory ${ }^{10}$ is too pessimistic $^{27}$ for very small crystallites, it now appears manyfold too optimistic!

Some details involved in the above points deserve amplification. The apparent difference between Glauber and Fujimoto rates of attenuation of $\Delta I / I$ with increasing scattering angle (Point 4) may, in some measure, be misleading. For very small crystallites the rings become increasingly blurred together as $s$ increases and, furthermore, the dynamic correction troughs deviate more from exact registry with the kinematic diffraction peaks. In Fig. 4 , the Glauber points used $\Delta I$ and $I$ values at their respective extremes rather than at a common $s$ value. Therefore, the meaning of $\Delta I / I$ at larger $s$ values is somewhat different for the Glauber plots than for the Fujimoto plots.

Points 2 and 3 taken together, and Fig. 3, give no clear indication that the Glauber single-double scattering corrections $\Delta I / I$ will ever link smoothly with the Fujimoto curves at larger $N$, where Fujimoto theory should become a much better approximation. Clearly, as $N$ increases, the single-single (kinematic) and single-double scattering terms become insufficient, and single-triple, double-double, and higher order terms begin to be needed. Clearly, also, as $N$ decreases, the Fujimoto approximations become less rigorous. There is no evidence that there is any range of $N$ in which the single-double scattering and the Fujimoto theories are both, simultaneously, reasonably good approximations. It is reasonable that, at larger $N$, higher order scatterings reduce $\Delta I$ just as $\Delta I$ (singledouble) reduces the interference peaks $I_{2}$.

Is there any evidence, then, that the Glauber theory at the single-double scattering level constitutes an adequate description for clusters, at least in the limit of small $N$ ? The answer seems to be yes according to studies of certain cases for which the Born approximation is tractable, because the two approaches were found to yield similar answers ${ }^{6,14}$ for the very small clusters treated (i.e., gas-phase molecules). Even more convincing is the agreement with experiment in the cases studied incluciing $\operatorname{ReF}_{6},{ }^{5,6} \mathrm{IOF}_{5},{ }^{7}$ and $\mathrm{XeOF}_{4} \cdot{ }^{7}$ How large $N$ can be before the single-double treatment is insufficient has not been established. Calculations including only single-double scatterings become rapidly encumbered with enormous numbers of terms as $N$ increases over 100. Calculations explicitly including higher order terms would become formidable. But the single-double scattering treatment, which warns of surprisingly large effects for $16 \AA$ gold clusters, must certainly be superior to the kinematic treatment usually applied and, in this range, more reliable than the conventional dynamic theory for crystals. For such minute crystallites conventional dynamic treatments are too difficult to carry out rigorously, particularly in the case of crystallites of arbitrary shape and arbitrary internal structure that can be handled in a straightforward way by the Glauber approach. In view of the increasing importance of electron diffraction studies of nucleation clusters, the application of an eikonal scattering theory may prove to be useful.

\section{ACKNOWLEDGMENTS}

We express our gratitude to the Laboratoire de Diffraction Électronique, Université de Paris-sud for congenial arrangements leading to the initiation of this 
research during the Visiting Professorship of one of us (L.S.B.) in the spring of 1973. We are pleased to acknowledge the Orsay Cluster Group for its contributions including fruitful discussions and computing facilities. The development of a computer code and numerical calculations were supported by C.N.R.S. The theoretical treatment was supported by a grant from the National Science Foundation.

*Institut d'Electronique Fondamentale, Laboratoire Associê au C.N.R.S.

${ }^{1} J$. Farges, B. Raoult, and G. Torchet, J. Chem. Phys. 59, 3454 (1973); J. Farges, M. F. de Feraudy, B. Raoult, and G. Torchet, J. Phys. (Paris) 36, C2 (1975); J. Farges, M. F. de Feraudy, B. Raouit, Ph. Schwartz, and G. Torchet, Acta Crystallogr. A 31, 171 (1975).

${ }^{2}$ F. Vergand, Philos. Mag. 31, 537 (1975).

${ }^{3} \mathrm{Ph}$. Buffat and J. P. Borel, Phys. Rev. A 13, 2287 (1976).

${ }^{4}$ See, for example, C. W. B. Grigson, in Advances in Electronics and Electron Physics, Supplement 4: Electron Beam and Laser Beam Technology, edited by L. Marton and A. B. El-Kareh (Academic, New York, 1968), pp. 189 and 199.

${ }^{5}$ E. J. Jacob and L. S. Bartell, J. Chem. Phys, 53, 2231 (1970).

${ }^{6}$ L. S. Bartell and T. C. Wong, J. Chem. Phys. 56, 2364 (1972); T. C. Wong and I. S. Bartell, ibid. 58, 5654 (1973). ${ }^{7} \mathrm{C}$. L. Ritz, doctoral dissertation, The University of Michigan, 1975, and unpublished research.
${ }^{8} \mathrm{H}$. Bethe, Ann. Phys, Leipzig 87, 55 (1928).

${ }^{9} \mathrm{C}$. H. MacGillavry, Physica (Utrecht) 7, 329 (1940). See also the reviews in J. Phys. Soc. Jpn. 17, Suppl. B II (1962).

${ }^{10}$ M. Blackman, Proc. R. Soc. London 173, 68 (1939).

${ }^{11}$ F. Fujimoto, Z. Naturforsch. Teil A 20, 367 (1965).

${ }^{12}$ H. Fengler, Z. Naturforsch. Teil A 16, 1205 (1961).

${ }^{13}$ L. S. Bartell, J. Chem. Phys. 63, 3750 (1975).

${ }^{14}$ R. A. Bonham and E. M. A. Peixoto, J. Chem. Phys, 56, 2377 (1972).

${ }^{15}$ A. C. Yates, J. Chem. Phys, 57, 1636 (1972).

${ }^{15}$ A. C. Yates and A. Tenney, Phys. Rev. A 5, 2474 (1972).

${ }^{17}$ P. J. Bunyan, Proc. Phys. Soc. London 82, 1051 (1963).

${ }^{1 /}$ J. Gjonnes, Acta Crystallogr。13, 1975 (1964).

${ }^{19}$ R. A. Bonham, J. Chem. Phys. 43, $1103(1965)$; J. W. Liu and R. A. Bonham, J. Mol. Struct. 11, 297 (1972).

${ }^{20}$ V. C. Romanov, A. V. Neymark, and M. G. Anashkin, Zhur. Khim. 15, 383 (1974).

${ }^{21}$ R. J. Glauber, Lect. Theor. Phys. 1, 315 (1959).

${ }^{22}$ Here, eikonal is used in the sense expressed by R. G. Newton, Scattering Theory of Waves and Particles (McGraw-Hill, New York, 1966), Chap. 18.

${ }^{23}$ G. Moliere, Z. Naturforsch. Teil A 2, 133 (1947).

${ }^{24}$ R. A. Bonham, Trans. Am. Cryst. Assoc. 2, 165 (1966).

${ }^{25}$ L. Schafer, A. C. Yates, and R. A. Bonham, J. Chem. Phys, 55, 3055 (1971).

${ }^{26} \mathrm{Z}$. G. Pinsker, Electron Diffraction (Butterworths, London, 1.953), Chap. 8 .

${ }^{27}$ Blackman's treatment, Ref. 10 , did in fact overestimate one factor; a value of $\left|v_{11}\right|^{2}$ was used which is twofold larger than the more modern value adopted in the present paper. 